\title{
The Semantic Study of Generics
}

\author{
Zhengling Fu \\ College of International Studies, Southwest University \\ Chongqing 400715, China \\ Tel: 86-135-9419-4776 E-mail: rainbow9119@163.com
}

Received: April 12, 2016 Accepted: April 25, 2016 Published: April 26, 2016

doi:10.5296/jsel.v4i1.9380 URL: http://dx.doi.org/10.5296/jsel.v4i1.9380

\begin{abstract}
This paper makes efforts to reveal cognitive motivation of generics, holding that generics and metonymy are the same in the aspect of categorization and generalization, that generics' economy expression and incomplete statements are closely related to human's innately given capacity to generalize from instance to type, or from subtype to type, and communicating economically with least efforts and least expression is universally accepted rather than the vise verse.
\end{abstract}

Keywords: Generics, metonymy, categorization, salience, activation 


\section{Macrothink}

\section{Realization of Generic NPs}

As the name suggests, generic sentences, in all languages, express generalization.

No known language contains a specific construction which is exclusively devoted to the expression of genericity (Dahl, 1975). Yet there is no language that does not express genericity in some form or another. In English, as in many other languages, genericity can be manifested through a variety of syntactic constructions. For example, despite their syntactically different subjects, all of the sentences in (1)-(6) are traditionally considered to be generic:

(1) Indefinite singular subject:

a. A whale is a mammal.

b. A blue whale eats plankton.

(2) "Bare plural" subject:

a. Blue whales eat plankton.

b. Blue whales are on the verge of extinction.

(3) Definite subject:

a. The killer whale is not dangerous to humans.

b. The rat reached Australia in 1770. (Krifka et al. 1995:12)

(4) Mass noun subject:

Wine contains at least $10 \%$ alcohol.

(5) Proper noun subject:

John speaks very quietly.

Notice that the same kind of NP subjects can appear in nongeneric sentences as well:

(6) a. A blue whale attacked our ship last week.

b. Blue whales are diving under our ship.

c. The whale is yours, if you want it.

d. Wine was spilled on my dress.

e. John was speaking very quietly now.

The semantic difference between the sentence in e.g. (1)-(5) and those in (6) is very strong. All the sentences in the first group express some kind of generalization. For example, the sentences in (1) and (2) do not talk about particular whales, but rather about whales or blue whales in general, or about the whole species of blue whales. Similarly (4) gives a property of wine in general (as opposed to (6d), which talks about a particular quantity of wine), and (5) talks about what John does in general (as opposed to (6e), which concerns what John did in a particular situation).

Genericity with nominal arguments is the most popular, and most essential. The following three groups of generic NPs are the concern of this dissertation, and the other is beyond the discussion: 


\subsection{Bare Plural Subjects}

The most common way to express a generic sentence in English is with a bare plural, i.e. a plural noun preceded by no determiner. It is well known that bare plurals may receive not only a generic reading, but an existential one as well. Thus, while (7a) makes a generalization about plumbers in general, ( $7 \mathrm{~b})$ states that there are some plumbers who are available.

(7) a. Plumbers are intelligent.

b. Plumbers are available.

What is the denotation of a generically interpreted bare plural? There are cases where the answer appears to be simple. Consider this typical example:

Dinosaurs are extinct.

There is no individual dinosaur that is extinct; individual dinosaurs are just not the sort of thing that can be extinct - only the kind dinosaur can have this property. A natural account for (8) is that it predicates the property of being extinct directly of the kind dinosaur. It follows, then, that the bare plural dinosaurs denotes this kind in (8). Krifka et al. (1995) refer to such sentences, which predicate a property directly of a kind, as cases of direct kind predication. They distinguish between them and those sentences, which predicate a property of instances of a kind, and not of the kind as a whole; these are named characterizing generics.

What is the denotation of a bare plural in a characterizing generic? Some researchers (e.g. Wilkinson 1991; Diesing 1992; Kratzer 1995) claim that bare plurals are ambiguous: they may denote kinds, in which case we get direct kind predication, or they may be interpreted as indefinites, i.e. as variables ready to be bound by the generic quantifier, resulting in characterizing generics.

Moreover, Carlson $(1977$; 1982) points out that generic bare plurals behave in a way that is similar to referring expressions, rather than indefinites or quantifiers. His arguments apply equally well to characterizing generics and direct kind predication. For example, he notes that if the antecedent of a pronoun is a name, it can replace the pronoun without a change in meaning; not so, in general, when the antecedent is an indefinite. Generics seem to behave like names, rather than indefinite, in this regard:

(9) a. Fred walked into the room. He smiled (= Fred smiled).

b. A man walked into the room. He smiled ( $\neq$ A man smiled).

c. Dogs are intelligent mammals. They are also man's best friend (= Dogs are man's best friend).

\subsection{Indefinite Singular Subjects}

Unlike bare plurals and definite singulars, indefinite singulars may not refer to kinds, as the unacceptability of the following examples indicates:

(10) a. *A giant panda is an endangered species.

b. *A dinosaur is extinct. 
The distribution of the indefinite singular is restricted compared with that of the bare plural, but in ways that are different from those of the definite singular. Consider the following pair (Lawler 1973):

(11) a. A madrigal is polyphonic.

b. *A madrigal is popular.

While (11a) receives a generic interpretation, (11b) cannot. In contrast, both (12a) and (12b) are fine.

(12) a. Madrigals are polyphonic.

b. Madrigals are popular.

Lawler (1973) claims that this difference between bare plural and indefinite singular generics is due to the fact that the latter are restricted to properties that are, in some sense, "necessary," "essential," "inherent," or "analytic." Thus, whereas polyphonicity is an essential property of madrigals, popularity is not, hence the unacceptability of (11b).

Krifka et al. (1995) propose an account of this phenomenon, based on the fact that indefinite singulars may not refer to kinds. They suggest that all cases where the indefinite singular generic is disallowed are cases of direct kind predication. That is to say, just like (8) expresses a property directly of the kind dinosaur, and not of individual dinosaurs, (12b) expresses a property directly of the kind madrigal. Specifically, unlike (12a), the logical form of (12b) does not involve the generic quantifier. Since indefinite singulars cannot occur in cases of direct kind predication, (11b) is ruled out.

\subsection{Definite Subjects}

Just like bare plurals, definite singular generics may occur in cases of direct kind predication as well as characterizing generics as exemplified by the following:

(13) a. The giant panda eats bamboo shoots.

b. The giant panda is an endangered species.

Sentence (13a) is about individual pandas, whereas (13b) is about the kind giant panda as a whole.

Generics with definite subjects usually take three forms : the + singular nouns ; the + plural nouns; the + adjectives. For example:

(14) The dog is man's first domestic animal.

When plural nouns nouns modified by definite articles expressing generalization, nouns can only refer to nationality or nation, for example :

(15) The Chinese are diligent.

(16) The Welshmen are fond of singing. (specific reference)

Not all nouns referring to nationality can be used to express generalization, especially those ended with -man, or -men, which frequently refer to specific reference, such as (16). While 
noun phrases consisting of definite articles and nouns ended with -ish, -sh or -ch can generally referred generically. Quirk et al. examplified this in A Comprehensive English Grammar for Foreign Students.

What's more, definite articles can modify adjectives to form plural generic noun phrases, indicating a group of people sharing a certain similarity, such as the rich, the poor. However, there exist a misunderstanding for this structure, see the following:

(17) The poor need more help.

(18) The poor people need more help.

"The poor" (17) is believed to be equivalent to "the poor people", this is not the case. In fact, the + people is a specific reference rather than a generic strcture, thereby, (17) is not equivalent to (18).

\subsection{Generic Quantifiers vs. Universal Quantifiers}

Being generalizations, generics have the property of tolerating exceptions. The initial instinct is to treat generics as universally quantified sentences. However, if you learn that Elena eats oatmeal for breakfast, she need not eat oatmeal at every breakfast. Or, the commonly found example Birds fly is tolerant of exceptional penguins, ostriches and other flightless birds. The limits of this exceptionality has proven extremely difficult to quantify - how long must Elena go without eating oatmeal for breakfast before the generalization no longer holds? How many flightless birds need there be in order for Birds fly no longer to be thought true? While some quantitative understanding of exceptionality plays a role, most researchers agree that generics requires an additional component of meaning, or a different arrangement of meaning altogether, to give an account of exceptionality.

The most commonly assumed semantic analysis of generics is outlined in Krifka et al. (1995). It posits an operator, which is often implicit in the linguistic form, which is a dyadic relation between the interpretations of two constituents partitioned from the sentence it is operating on, a "restrictor" and a "matrix" or "nuclear scope", in keeping with the most commonly accepted semantic analysis of quantification. As this dyadic operator is focus-sensitive, generic sentences can be ambiguous according to which constituent meaning is assigned to the restrictor and matrix. For example, Milsark (1974) notes the ambiguity of the sentence Typhoons arise in this part of the Pacific. As discussed in Carlson (1982), if the subject noun phrase typhoons is understood as the restrictor, and the predicate of the sentence the matrix, then the interpretation assigned is akin to asserting that generally speaking, if something is a typhoon, it then arises in this part of the Pacific ocean (and not elsewhere). If, on the other hand, in this part of the Pacific is assigned to the restrictor, then the resulting interpretation is that, in this part of the Pacific, there arise typhoons (from time to time), and perhaps elsewhere as well. Word order in English and other languages can affect how the sentence is partitioned by this and other focus-sensitive operators (Diesing, 1992 discusses German at some length). For instance the English sentence In this part of the Pacific arise typhoons has only the latter of the two readings. Krifka et al. (1995) describes the generic operator as a "default quantifier" in order to account for exceptionality and intensionality. Other 
researchers take a different approach, such as modifying possible worlds to enrich the interpretive structure with a notion of "normality" or "prototypicality" (e.g. Eckardt, 1999; Heyer, 1985). The basic idea here is that one can reduce the generic operator to a universal statement relativized only to the most typical or normal individuals of the domain, or to "normal worlds". Cohen (1999) suggests that the generic operator is a quantificational operator similar in contents to "most", though relativized to a partition of individuals and situations that is pragmatically driven, and not determined by the focus structure of the sentence. For instance, in asserting that mammals bear live young, one is partitioning the set of mammals by gender and age, as only mature (fertile) females have such capability. According to this understanding then a generic is information assumed to hold for any given relevant instance, unless specific information is given otherwise.

\section{Metonymy, Generics and Categorization}

Generic statements are usually understood as making reference to kinds of things. It is now commonly accepted that reference is not only limited to individuals or pluralities of individuals, but also to kinds or types of things as well. When we speak of "kinds", we intend a classification system that is based on the denotations of nominal expressions of the language. The mental process of classification is commonly called categorization, and its product is cognitive categories.

Generic level is also called basic level in psychology, and its significance can be further summarized as the following:

i. The most salient difference between objects and organisms appear in the basic level.

ii. Members of basic level categories have shape in common.

iii. Members of basic level categories have motor movements in common.

iv. Basic level names are used to identify objects.

v. Basic level names produce the priming effect.

Not only our language but also our thoughts are both structured by metonymic concept, which is, like metaphor, a widely used way of thinking. The structure of categories can present the conceptual nature of metonymy clearly, since metonymy mainly involves mapping within the same category, relevant categories or across different levels of categorization. A category can be represented by the most representative members of the category, termed as prototypical members, and the process is called prototypical effects. The example of "housewife mother" can properly indicate this point: people tend to think of the MOTHER category in terms of "housewife mother". When the word "mother" is spoken of, the image of stereotypical subcategory "housewife mother" automatically appears in people's mind. Such subcategories of mother as "adaptive mother, stepmother, and unmarried mother" do not occur frequently in people's mind unless in certain specific cases, since they are much less typical members compared with "housewife mother". Therefore, the category MOTHER is typified by typical members of this category or the stereotypical subcategory "housewife mother". People have no doubt to conclude that basically all categories have metonymic 
structure considering most categories have prototypical structure. To further the study, it is necessary to have a look at the different levels of categorization, since prototype categories are most fully developed on the basic level. According to Rosch's findings, the hierarchical structure of language is constituted by superordinate category, basic-level category and subordinate category, among which basic-level category is the most important one.

The hierarchy of categorization and prototypical structure can exert profound influence on the truth judgment of generics. Rosch et al. (1976) have identified in hierarchical representations a special level, which they call the basic level. For example, dog is a basic level concept, while mammal is a superordinate and cocker spaniel is a subordinate. Members of basic level categories have a significant number of properties in common, and are relatively easily distinguished from members of other basic categories. "This means that when we observe the environment, the first categorization is made at the basic level (e.g. This is a dog, rather than this is a mammal or this is a cocker spaniel)." In 1976, Rosch et al. have discovered a number of properties that distinguish basic level categories in regard to superordinate and subordinate categories.

Rosch et al. suggest that shape can distinguish basic level concepts form one another but cannot distinguish subordinates. Shape is a candidate partitioning criterion to divide a superordinate concept into basic level concepts, but not if we further partition a basic level concept into its subordinates. Here, for example, is part of the representation of fruit (basic level concepts are in bold):

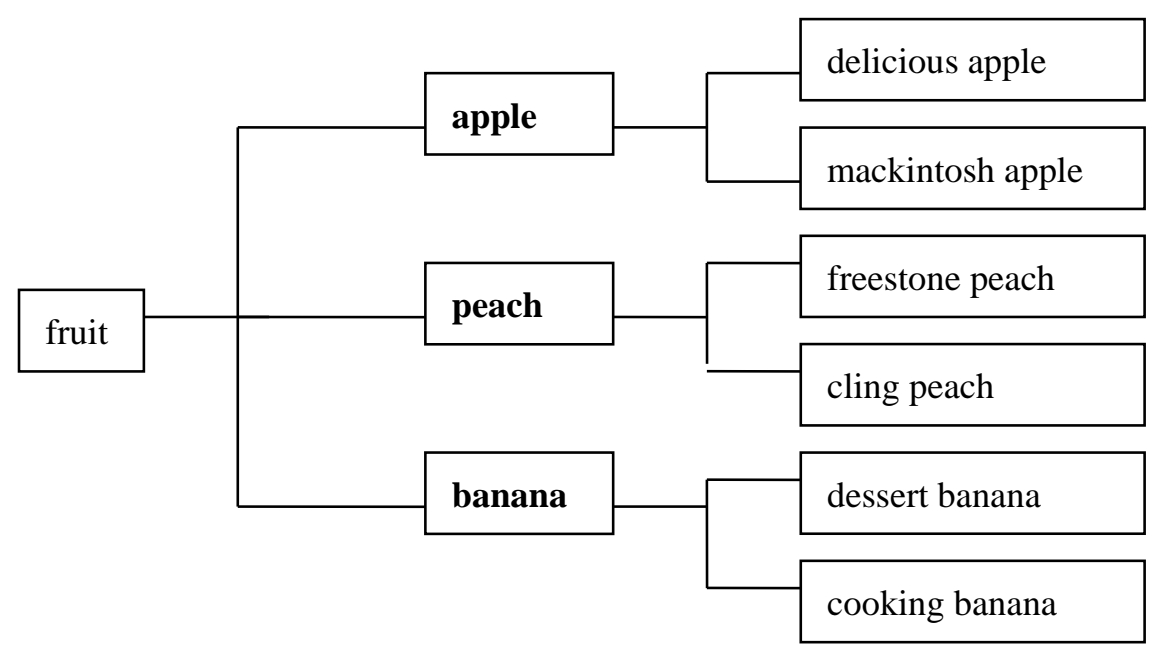

Figure 1. The hierarchy of fruit categorization

Note that the basic level concepts, apple, peach, and banana, differ in their shapes; in contrast, the subordinate of any one of them (e.g. delicious apple and mackintosh apple) have very similar shapes. Therefore, we would make the following prediction: if a property involving shape is predicated of a superordinate (e.g., fruit) in a generic sentence, then the existence of even one basic level concept that does not satisfy the property would suffice to violate homogeneity (for a generic sentence to be true, its domain needs to be homogeneous with 
respect to salient partitions ). On the other hand, if some shape is predicted of a basic level concept, homogeneity would be satisfied, and the sentence would be acceptable. Anything subordinate to basic level concepts cannot be partitioned by shape, so if a shape is predicated of a subordinate, the sentence would also be fine.

(19) ? Fruits are round.

(20) Apples are round.

(21) Delicious apples are round.

In the above group, sentence (19) is not acceptable, despite the fact that most fruits are round, because shape may partition the superordinate concept FRUIT into basic level concepts, for example, some fruits (e.g., apple) are round, and some (e.g., banana) are not.

Even though all apples are not round (some are oblong), sentence (20) is legitimate. The reason is that, shape cannot further partition a basic level concept APPLE. Thus, homogeneity is satisfied, and (20) is acceptable (and true). Of course, delicious apple, too, being a subordinate level, may not be partitioned according to shape, hence (21) is fine.

It is reasonable to assume that not only shape, but other perceptually salient properties behave in the same way: basic level concepts may not be partitioned according to perceptually salient properties. We would predict, then, that generics that predicate a perceptually salient property of a basic level concept will not violate homogeneity. This does, indeed, appear to be the case.

(22) a. Roses are red.

b. Tigers have stripes.

Although there are certainly white and yellow roses, (22a) is fine. Since rose is a basic level concept, if we assume that basic level concepts cannot be partitioned into their subordinates based on color, this judgment would thereby be explained.

Sentence (22b) is also acceptable (and true), despite the existence of albino tigers. Since tiger is a basic level concept, and having stripes is a perceptually salient property, this judgment is precisely as predicated. And, of course, (23) is also fine, since Bengal tiger is a subordinate of tiger.

(23) Bengal tigers have stripes.

Tversky and Hemenway (1984) have proposed an additional property of basic level concepts. They claim that superordinates may be partitioned according to their parts, but basic level concepts may not. The following representation of furniture, from Rosch et al. (1976) can best illustrate this: 


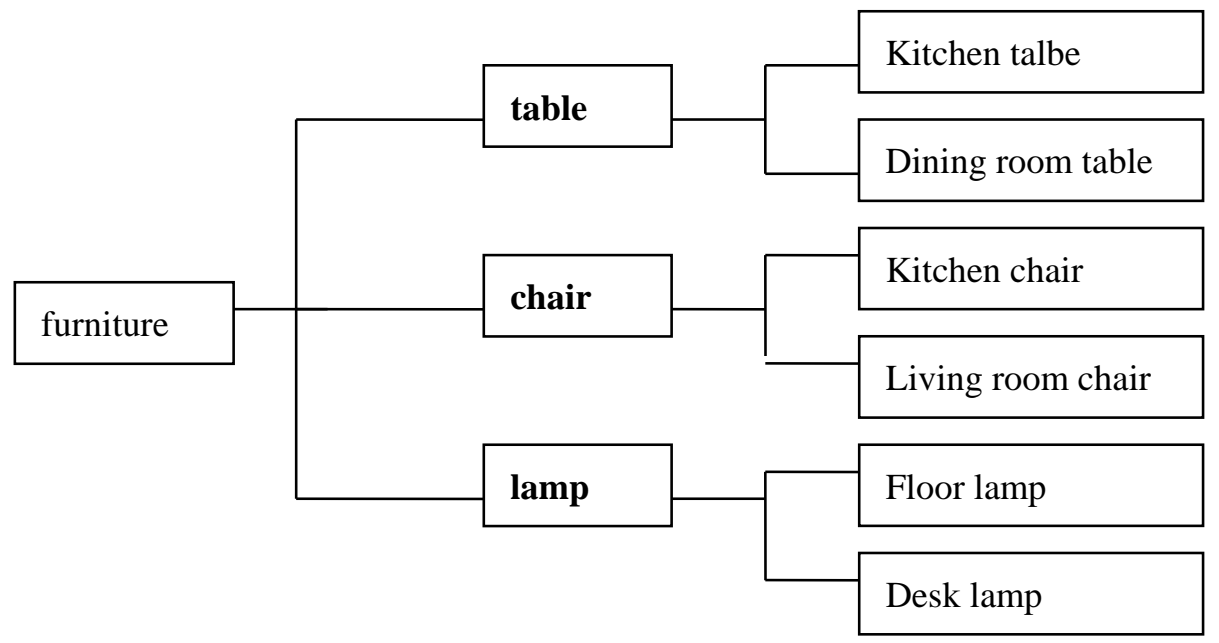

Figure 2. The hierarchy of furniture categorization

The basic level concepts table, chair, and lamp can be differentiated from the parts they have. For example, tables have tops, chairs have backs, and lamps have light bulbs. But the subordinates do not differ in the same way: kitchen tables and dining room tables have the same parts essentially.

Therefore, we predict that if a generic predicates of a superordinate level concept the property of having a certain part $\mathrm{p}$, any basic level concept that does not have $\mathrm{p}$ would suffice to violate homogeneity. In contrast, homogeneity should be satisfied, if having $\mathrm{p}$ is predicated of a basic level concept (or its subordinate). This is really the case in point:

(24) a. ? Furniture has four legs.

b. Tables have four legs.

c. Coffee tables have four legs.

As a superordinate level concept, furniture may be partitioned according to its parts. Since some piece of furniture (e.g. lamp) does not have four legs, homogeneity is not satisfied and the sentence is invalid. In contrast, table may not be partitioned according to the parts it has. Although some tables do not have four legs, they do not form a class into which the basic level concept may be decomposed. Rather, these tables are spread more or less evenly among the subordinates of table. Hence, homogeneity is satisfied, and the sentence is fine. The same holds for the subordinate coffee table, accounting for the acceptability of (32c).

The same phenomenon can be applied to biological taxonomies, where the basic level is higher in the hierarchy; for example, Rosch et al. have discovered that it is the category bird itself rather than species of birds(e.g., robin, duck, etc.) that are at the basic level. Actually, there is a difference between (33a) and (33b).

(25) a. ? Animals have legs.

b. Birds have wings.

c. Ducks have flat bills. 
Since animal is a superordinate level concept, it may be partitioned with regard to its parts. Many animals have legs, but some do not; hence, homogeneity is violated, and (25a) is bad. In contrast, (25b) is fine, although not all birds have wings.

Now consider the following generic sentences:

Mosquitoes carry the West Nile Virus.

Sharks attack bathers.

Pittbulls maul children.

Tigers eat people.

These generics are intuitively true, yet every few members of the kind in question actually possess the predicated property. When dealing with the sort of information that one would be well-served to be forewarned about, the mechanism generalizes in the face of even just a few members of the kind possessing the property.

Of course, generalizing such information to every kind that has a member with the property would be inefficient. We do not consider "animals carry the West Nile Virus" or even "insects carry the West Nile Virus" to be true, even though both kinds have some members that carry the virus, since those virus-carrying mosquitoes belong to both kinds. Genericity in basic level can avoid generalization too broadly.

\section{Relative Salience}

Unlike metaphor, metonymy involves a relation of contiguity and salience. Since in communication, people tend to use the most salient aspect of an object, idea, or event to refer to the object, idea, or event as a whole, the understanding of metonymy must mainly involve the recognition of salient part of an object, idea, or event as a whole. According to Langacker (1991), the highly salient entity serves as a cognitive reference point to evoke another unsalient entity, speaker and writer (or listener and reader) use the background in the concept provided by other parts to contact conceptually with another conceptual entity. The reference point, as the salient part in the discourse, thus built a context in which speaker and writer (or listener and reader) get the conceptual access with other unsalient conceptual entities in the context. In conceptual metonymy THE FACE FOR THE PERSON, exampled as Here comes a big nose, the source meaning a big nose, serves as the vehicle, relatively salient, to activate the target meaning "a person who has a big nose". Take another example, a typical generic sentence "Birds fly" to illustrate it further. With many features from different taxonomy, it is true that birds eat, birds drink and etc., however, the most salient feature or the feature which first comes into our mind at the mention of birds is "the ability of flying", which can distinguish birds from other animals. Being asked to describe birds with several words, "flying" must be in the list, since it is a relatively salient feature.

\section{Activation}

We have found support for activation in cognitive psychology. In the spreading activation theory, activation starts at a node or a concept, the more closely related another node or concept is, the more likely it is to be activated. Activation plays an irreplaceable role in the 
generation and understanding of generics.

When one uses the notions of ICM as a theoretical background, as Radden \& Kovecses (1999) do, it is still possible to claim that with the metonymy mechanism, a (sub)domain is mentally activated via another (sub)domain in the same ICM or frame. The intradomain activation of one (sub)domain by another is an essential ingredient of metonymy. Once the mechanism has identified a characteristic dimension for a domain of kinds, the value along the dimension for a given kind within the domain will be activated, and if the activated correspond to the sentence, then truth is realized even if in face of exceptions. For example, chickens lay eggs, this sentence is intuitively true without any doubt, even if there exist some exceptions to itcocks do not lay eggs. In such sentence, the domain of reproduction is activated, and then this sentence is to seek to fill in a value along reproduction. Since reproduction only involves female part of the animal kind, so the cases of male parts do not influence the truth of the sentence. And of course, if we can find a chicken which produce live young, then this sentence is to be false. Some researches hold that generics' truth condition is hard to define, with the challenge of another sentence "chickens are female". "Chickens lay eggs" is true, while "chickens are female" is false. What makes the difference? The answer lies in the fact that the second sentence activates the domain of gender. The animal kind "chicken" includes two genders, male and female, so if the second sentence is true, then male chickens are neglected. The domain activated is profoundly essential in judging the truth of generics.

\section{Conclusion}

The metonymic mechanism, basically cognitive mechanism, is responsible for generation of genericity. ICMs serve to restrict the generic referent to characteristic dimensions, or essential and salient property of a certain type, which is actually in the metonymic model INSTANCE FOR TYPE or SUBTYPE FOR TYPE. Genericity is conceptual metonymy because within the same idealized cognitive model, generic sentence provides mental access to the target type by instances or subtype. What generics correspond to is characteristic dimensions or essential property instead of actuality and thus accounts for the fact that generic reference usually allows for exceptions.

\section{Acknowledgement}

This research is financed by The Social Science Planning Project of Chongqing (2015YBYY134), The Higher Education and Teaching Reform Project of Chongqing (153030), The Teacher and Teaching Development Project of Southwest University (SWFZ20150009) and The Fundamental Research Funds for the Central Universities (SWU1509176).

\section{References}

Carlson, G. (1977). Reference to Kinds in English. University of Massachusetts at Amherst dissertation. Also published 1980, New York: Garland.

Carlson, G. (1982). Generic terms and generic sentences. Journal of Philosophical Logic, 11, 145-181. 


\section{Macrothink}

Cohen, A. (1999). Think Generic! The Meaning and Use of Generic Sentences. Chicago: The University of Chicago Press.

Dahl, O. (1975). On generics. In E. Keenan (ed.), Formal Semantics of Natural Language. Cambridge: Cambridge University Press.

Eckardt, R. (1999). Normal objects, normal worlds and the meaning of generic sentences. Journal of Semantics, 237-278.

Heyer, G. (1990). Semantics and knowledge representation in the analysis of generic descriptions. Journal of Semantics, 7, 93-110.

Krifka, M., F. J. Pelletier, G. Calson, A. ter Meulen, J. Link, \& G. Chierchia. (1995). Genericity: an introduction. In G. Calson, \& F. J. Pelletier (eds.), The Generic Book. The University of Chicago Press, Chicago.

Langacker, R. (1991). Foundations of Cognitive Grammar. Vol.II. California: Stanford University Press.

Lawler, J. (1973). Studies in English generics. University of Michigan Papers in Linguistics $1: 1$.

Milsark, G. (1974). Existential Sentences in English. Ph. D. Dissertation. Massachusetts Institute of Technology. Distributed by Indiana University Linguistics Society. Chicago.

Radden, G., \& Z. Kovecses. (1999). Towards a theory of metonymy. In K-U. Panther \& G. Radden (eds.). Metonymy in Language and Thought. Amsterdam/ Philadelphia: John Benjamin's Publishing Company.

Rosch, E., \& B. Lloyd. (1976). Cognition and Categorization. Hillsdale, N.J.: Erlbaum Associates.

Wilkinson, K. (1991). Studies in the semantics of generic noun phrases. Ph.d. dissertation. Amherst: University of Massachusetts.

\section{Copyright Disclaimer}

Copyright for this article is retained by the author(s), with first publication rights granted to the journal.

This is an open-access article distributed under the terms and conditions of the Creative Commons Attribution license (http://creativecommons.org/licenses/by/3.0/). 